\title{
Evaluation of Outcomes of a Pharmacist-Run, Outpatient Insulin Titration Telepharmacy Service
}

Katharine Stockton, PharmD and Crystal Deas, PharmD, BCPS

Samford University, McWhorter School of Pharmacy

\begin{abstract}
Purpose: Diabetes can pose a significant disease burden for patients and is often challenging to manage in underserved patient populations with limited access to care. A pilot study was conducted to determine the impact of a pharmacist-run insulin titration service, provided via telephone, to patients of a local ambulatory care clinic with a large medically, underserved patient population. The pilot service was implemented in Spring 2018 at Cooper Green Mercy Health Services (CGMHS) and was provided by an affiliate clinical pharmacist who is also on faculty at Samford University's McWhorter School of Pharmacy. Patients who received care within the CGMHS diabetes clinic were eligible for referral to the service. The service was provided via a collaborative practice agreement. The purpose of this study was to evaluate and compare clinical outcomes of patients who received the telepharmacy service versus the standard of care. Standard of care was defined as patients whose insulin therapy was managed solely by the primary care provider or by a diabetes clinic provider, without clinical pharmacist involvement in the patient's care.

Methods: This manuscript presents the results of a retrospective chart review conducted at CGMHS of patients, ages 19 or older, with a documented diagnosis of type 1 or type 2 diabetes who received care during the timeframe of February 2018 through September 2018 - the initial months of the pilot telepharmacy service.

Results: Sixty-seven patients met criteria for inclusion in the analysis - 16 managed in the telepharmacy service, 28 in diabetes clinic, and 23 in primary care. Patients in the telepharmacy group achieved a mean A1c change of $-1.14 \%$ compared with $-0.88 \%$ in the diabetes clinic group and $+0.21 \%$ in the primary care group ( $p=0.061)$. In the telepharmacy group, $43.75 \%$ of patients experienced at least a $1 \%$ reduction in A1c from baseline compared with $35.71 \%$ in the diabetes clinic group and $26.09 \%$ in primary care ( $p=0.51$ ).

Conclusion: Integration of the clinical pharmacy services for insulin titration positively affected patients' degree of glucose control. Although no statistically significant reductions in A1c were observed in this study, it should be noted that pharmacist intervention was associated with a modestly higher percent A1c reduction from baseline vs. the standard of care. The pharmacist-run service produced changes in clinical outcomes that numerically exceeded those experienced by patients receiving specialty care, in the diabetes clinic, and within primary care. As a result of this study, the pilot program has remained in effect and is in the initial stages of expansion of the consult service to eligible primary care patients.
\end{abstract}

Key words: telepharmacy; insulin titration; pharmacist

\section{INTRODUCTION}

Diabetes is the seventh leading cause of death in the United States; leading to over 14.2 million emergency department visits in 2015. ${ }^{1}$ The diagnosis of diabetes is associated with a significant disease burden for patients, with the requirement of close monitoring and often frequent medication adjustments. Additionally, the condition is associated with several complications such as kidney damage, neuropathy, vision loss and cardiovascular complications when poorly managed. Diabetic patients often need individualized therapy, therefore demonstrating the key role of an interdisciplinary team.

Corresponding author: Crystal Deas, PharmD, BCPS Assistant Professor

Samford University, McWhorter School of Pharmacy

Email: cdeas@samford.edu
Lower socioeconomic status is associated with poor selfmanagement behaviors often resulting in poor glycemic control. ${ }^{2,3}$ Poor self-management can be due to a number of reasons, such as misunderstanding of how to manage diabetes, decreased access to care, and limited transportation. Previous studies have evaluated the use of telepharmacy services to manage diabetes in low-income populations, showing an increased access to care has an impact on glycemic control in diabetes. ${ }^{4}$

Cooper Green Mercy Health Services (CGMHS) is a countyoperated, outpatient healthcare center within the metropolitan Birmingham area that provides ambulatory care services, both primary and specialty care, for residents through use of a subsidized care model. The center serves a financially disadvantaged patient population who are adversely impacted by resource limitations that influence health - such as transportation issues, food insecurity, low health literacy, poor medication adherence, and limited disease self-management skills. In February 2018, Samford University's McWhorter School of Pharmacy collaborated with 
CGMHS to implement pharmacy services within the diabetes clinic. The service was provided at no cost to patients and was developed to increase access to care for those patients who were unable to reliably return to clinic for in-person visits but required close diabetes follow-up with frequent insulin adjustments.

A key component of the pharmacy service intervention was the initiation of an insulin titration service pilot, which was provided via telepharmacy for referred clinic patients. Given the pilot nature of the intervention, during the initial months of the service, only diabetes clinic providers served as referring providers for patients to receive the telepharmacy service. The diabetes clinic providers referred patients who had selfidentified transportation difficulties and, in the provider's professional judgement, had an anticipated need for frequent insulin adjustments. Those patients referred to the service were initiated on an insulin regimen by his/her CGMHS diabetes clinic provider prior to receiving the telepharmacy intervention. Under the care of a clinical pharmacist, patients were provided medication education and frequent telephone adjustments of insulin, as clinically indicated, per an established protocol. The process of referral and care within the service is depicted in Figure 1.

The purpose of this study was to review clinical outcomes of patients receiving the telepharmacy service versus the standard of care. In this study, standard of care was defined as patients whose insulin therapy was managed either solely by the patient's primary care provider or by a diabetes clinic provider, without clinical pharmacist involvement in the patient's care.

\section{METHODS}

\section{Study Design}

This study was a retrospective chart review. Data was collected from patients with a documented outpatient encounter during the timeframe of February 2018 through September 2018 which was the initial 8 months of the pilot intervention. Patients included were 19 years of age or older, had a documented diagnosis of type 1 or type 2 diabetes, had a documented prescription of insulin within the medical record during the study timeframe, had at least two documented outpatient encounters in the medical record, and a documented baseline and follow-up glycated hemoglobin (A1c). Medical charts were reviewed from eligible patients who received insulin management within three services: (1) telepharmacy; (2) diabetes clinic - managed by a provider other than a clinical pharmacist; and (3) primary care.

Patients from the telepharmacy group were identified utilizing a convenience sample of those who received care during the pilot. Of the 23 patients referred to the telepharmacy service, 16 returned for follow-up A1c lab monitoring within the study period and met inclusion criteria. Patients within the standard of care groups were identified by random selection via use a random number generator and report from the electronic health record (EHR) of patients seen in either primary care or diabetes clinic during the study period. One-hundred eight patient charts were identified through randomization and reviewed. Fifty-one patients met inclusion criteria for this analysis - 28 who were managed in diabetes clinic and 23 within primary care.

The primary outcome of the study was the difference in percent $A 1 c$ change among patients in the telepharmacy group compared to two defined standard of care groups. Secondary outcomes included percent compliance with recommended A1c monitoring (i.e., checked every 3 to 4 months) per ADA recommendations, percent of patients who obtained an absolute A1c value of less than $7 \%$, and percent of patients experiencing at least a $1-2 \% \mathrm{~A} 1 \mathrm{c}$ reduction between groups. ${ }^{5}$

\section{Statistics}

Statistical analyses were conducted using SPSS (IBM Corp. Released 2017. IBM SPSS Statistics for Windows, Version 25.0. Armonk, NY: IBM Corp). Chi-square was utilized for analysis of nominal data. For cases where a cell had $n<5$, the Fisher's Exact test was used. ANOVA was used to compare the means of the three groups, and the Independent t-test was utilized for sub-analysis comparison of means between two groups. An alpha value of $p<0.05$ was considered significant. The Samford University Institutional Review Board approved this research.

\section{RESULTS}

A total of 67 patients met criteria for inclusion in the analysis (Figure 2). The majority (86.57\%) of patients in the study were diagnosed with type 2 diabetes mellitus. Baseline characteristics were similar between groups and are described in Table 1.

More active management of insulin was noted in the telepharmacy group with the majority of patients in this group $(87.5 \%, n=14)$ having adjustments made to their insulin regimen during the study period vs. $75 \%(n=21)$ of patients managed in diabetes clinic and $39.1 \%(n=9)$ in the primary care group. The majority of patients in the telepharmacy group $(87.5 \%, n=14)$ were prescribed both a basal and bolus insulin. Whereas, $71.4 \%(n=20)$ of patients from the diabetes clinic group and $69.6 \%(n=16)$ from the primary care group were on combination basal and bolus insulin during the study period.

Patients in the telepharmacy group achieved a mean A1C change of $-1.14 \%$ compared with

$-0.88 \%$ in the diabetes clinic group and $+0.21 \%$ in the primary care group $(p=0.061)$ (Figure 3$)$. Secondary outcomes were also compared between groups (Table 2). In the telepharmacy group, $43.75 \%$ of patients experienced at least a $1 \%$ reduction in A1c from baseline compared with $35.71 \%$ in the diabetes clinic group and $26.09 \%$ in primary care $(p=0.51)$. There were no patients in the telepharmacy group who achieved an A1c reduction to below $7 \%$ in this study. However, it should be 
noted that the mean baseline A1c of patients in the pharmacy group was $10.56 \%$ compared to $9.44 \%$ in both of the standard of care groups (diabetes clinic and primary care, respectively). A post-hoc analysis of change in A1c revealed that $43.48 \%$ of patients in the primary care group, $25 \%$ of patients in diabetes clinic group, and $12.5 \%$ of patients in the telepharmacy group experienced an increase in A1c from baseline during the study period.

The majority of patients (68.75\%) in the telepharmacy group had an A1c rechecked and documented quarterly, per the American Diabetes Association recommendations, compared to $53.57 \%$ managed in diabetes clinic and $17.39 \%$ in primary care $(p=0.003) .{ }^{5}$ When the rates of A1c monitoring, per ADA recommendations, was compared between the telepharmacy vs. diabetes group and telepharmacy vs. primary care group in a sub-analysis, there was no significant difference noted ( $p>0.05$ for both comparisons). A statistically significant difference was noted in the sub-analysis when compliance with A1c monitoring was compared between diabetes clinic and the primary care group $(p=0.013)$.

\section{CONCLUSION}

In this study, the telepharmacy intervention resulted in a larger percent reduction in $\mathrm{A} 1 \mathrm{c}$ from baseline. However, a statistically significant difference was not noted between groups. While not a statistically significant finding, the results are clinically significant since the primary care group experienced an increase in A1c during the study period. This is an important observation, considering diabetes is typically managed by primary care providers in the United States.

While this study is not the first study to compare a pharmacistled diabetes intervention to standard practice for diabetes management, it is the first to our knowledge to compare clinical outcomes of insulin management via telepharmacy vs. primary care and diabetes clinic management in a primarily indigent patient population. A study conducted in a rural area of North Carolina assessed a clinical pharmacist's role on telehealth team, and noted a significant reduction in weight, A1c and LDL within the 12 months of the pharmacy intervention. ${ }^{6}$ However, when outcomes of the patients included in the telehealth group were compared to those managed in a clinic setting at an academic medical center, the change in \% A1c from baseline was non-inferior and did not demonstrate statistical or clinical significance. ${ }^{6}$ A similar observation of lack of statistical significance was noted in our study. However, in our study, the observation that the absolute \% change in A1c was numerically higher in the telepharmacy group vs. standard of care may suggest clinical significance. A study conducted by Pitlick et al. with a patient population similar in size and demographics to our study also compared outcomes at 6 months of patients enrolled in a pharmacist managed insulin titration service vs. standard of care, defined as patients managed by a primary care provider. ${ }^{7}$
A statistically and clinically significant reduction in A1c from baseline was noted at 6 months between the intervention and standard of care group. ${ }^{7}$ It should be noted that this study did not utilize telepharmacy as their primary method for insulin titration and there was only one defined standard of care group. Similarly, a study conducted at the St. Louis County Department of Health assessed \% A1c change at 3, 6, 9, 12 and 18 months, and study conclusion. ${ }^{8}$ Two groups were compared in the analysis - patients managed in the pharmacist-run insulin titration program and patients managed solely by their primary care provier. $^{8} \mathrm{~A}$ statistically significant difference in A1c at all study time points was noted between the pharmacy and standard of care group. ${ }^{8}$ A study conducted by WeidmanEvans et al. evaluated the impact of a pharmacist-led insulin telepharmacy service utilizing faculty pharmacists, similar to pharmacist resources in our service, within a family medicine clinic. ${ }^{9}$ The faculty followed patients by phone and made adjustments via a two-step process: (1) use of EHR to notify patients' providers of the pharmacists' recommended changes to insulin and (2) subsequent notification of patients on the insulin adjustments, following provider approval. ${ }^{9}$ The authors noted a typical 3-4 hour delay after sending EHR alerts to initiating follow-up contact with patients to educate on the insulin dose change. ${ }^{9}$ The researchers noted a decrease in A1C from baseline of $1.55 \%{ }^{9}$, which is similar to the reduction noted in our study. No comparisons were made to a standard of care group in the Weidman-Evans study; real-time adjustments were not made to insulin, as in our study. Given the telephonic delivery of the pharmacy intervention and the indigent nature of our clinic's population, which increases the likelihood of frequent interruptions in accessible phone service, providing care during one point of contact is preferred.

Not only is our study is innovative in that it evaluates outcomes of an insulin telepharmacy service in comparison to two standard of care groups, it also evaluated the difference in adherence to ADA guideline-recommended A1c monitoring between groups. A significant difference between groups was noted in percentage of patients who received A1c monitoring at the recommended intervals. However, when groups were compared separately in a sub-analysis, there was no statistically significant difference noted between the telepharmacy group vs. primary care and telepharmacy vs. diabetes clinic patients but rather was noted between the diabetes clinic vs. primary care patients. As reimbursement models have shifted to value-based care with a focus on clinical metrics, it is important for pharmacy services to track the impact of interventions on adherence to guideline recommendations that are associated with quality metrics.

Our study has important limitations. A convenience sample was utilized for identification of patients in the telepharmacy group and thus limits generalizability of results. The sample size was small. A high "no show" rate was observed in patients evaluated for inclusion in the study, which attributed to the 
limited sample. Additionally, the small pilot was conducted in a single center, which may limit external validity.

The results from this study suggest that innovative ways to provide care for patients, such as through telepharmacy, poses a promising solution to patients limited transportation resources. $^{6}$ Results could be used to support the implementation of a similar pharmacist-run insulin titration service in other ambulatory care settings. Given the study findings, the telepharmacy service has remained in effect at the clinic with plans to expand the consult service to eligible patients within the primary care clinic.

\section{Conflict of Interest: None}

\section{REFERENCES}

1. National Diabetes Statistics Report, 2017 Estimates of Diabetes and Its Burden in the United States. CDC National Diabetes Statistics Report. March 2018:1-20. https://www.cdc.gov/diabetes/pdfs/data/statistics/nati onal-diabetes-statistics-report.pdf

2. Norris SL, Engelgau MM, Venkat Narayan KM. Effectiveness of self-management training in type 2 diabetes: a systematic review of randomized controlled trials. Diabetes Care. 2001;24:561-87.

3. Houle J, Lauzier-Jobin F, Beaulieu M-D, et al. Socioeconomic status and glycemic control in adult patients with type 2 diabetes: a mediation analysis. BMJ Open Diabetes Research \& Care. 2016;4(1):1-8.

4. Levy N, Moynihan V, Nilo A, et al. The Mobile Insulin Titration Intervention (MITI) for Insulin Adjustment in an Urban, Low-Income Population: Randomized Controlled Trial. J Med Internet Res. 2015;17(7):e180.

5. 6. Glycemic targets: Standards of Medical Care in Diabetes-2018. Diabetes Care 2019. American Diabetes Association. Diabetes Care. 2019;42(Suppl. 1):S61-S70.

6. Nye AM. A Clinical Pharmacist in Telehealth Team Care for Rural Patients with Diabetes. North Carolina Medical Journal. 2017;78(3):183-184.

7. Pitlick JM, Brooks AD. Glycemic Control in PharmacistManaged Insulin Titration Versus Standard Care in an Indigent Population. Diabetes Spectrum. 2011;24(4):211-217. doi:10.2337/diaspect.24.4.211.

8. Salvo MC, Brooks AM. Glycemic Control and Preventive Care Measures of Indigent Diabetes Patients Within a Pharmacist- Managed Insulin Titration Program vs Standard Care. Annals of Pharmacotherapy. 2012;46(1):29-34. doi:10.1345/aph.1q512.

9. Weidman-Evans E, Evans J, Eastwood R, Fort A. Implementation of a pharmacist-run telephonic insulin titration service. J Am Pharm Assoc. 2012; 52(6):e26672. 
Figure 1. Telepharmacy Intervention

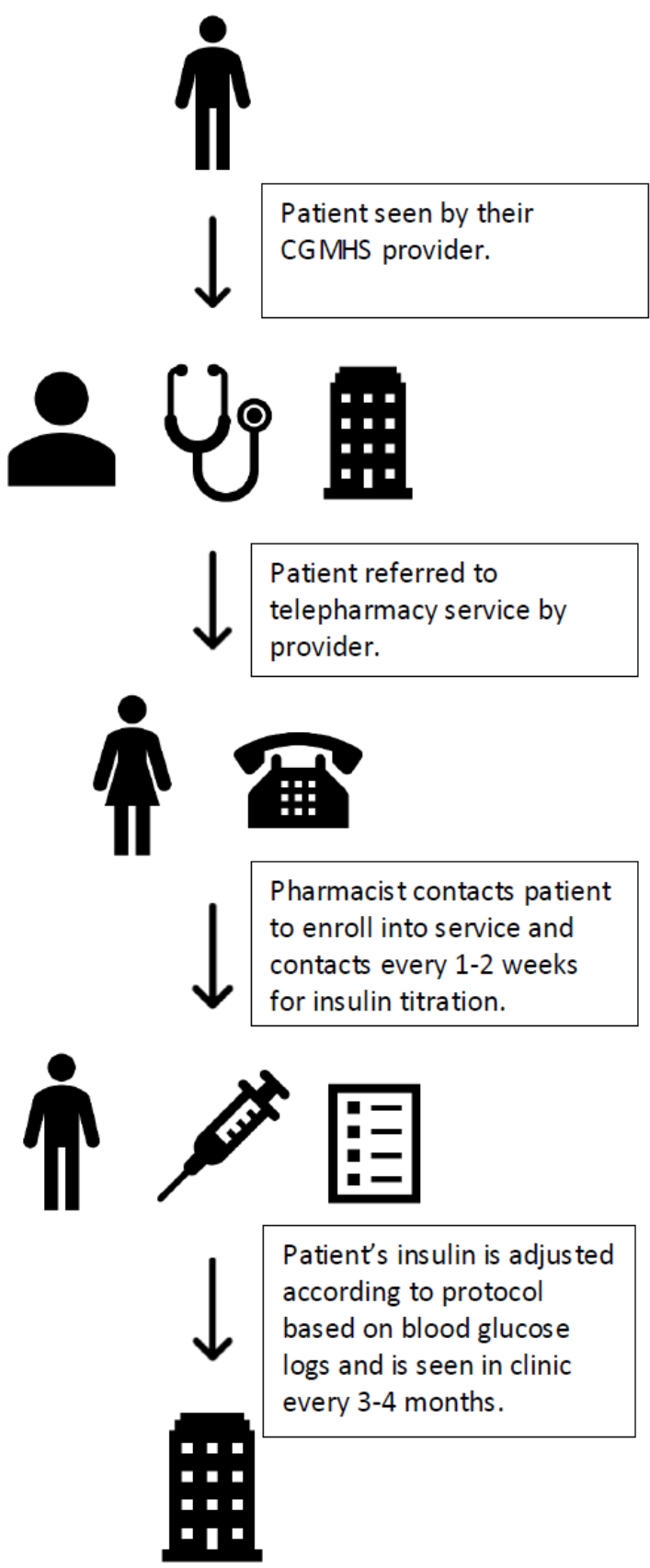


Figure 2. Patient allocation by group

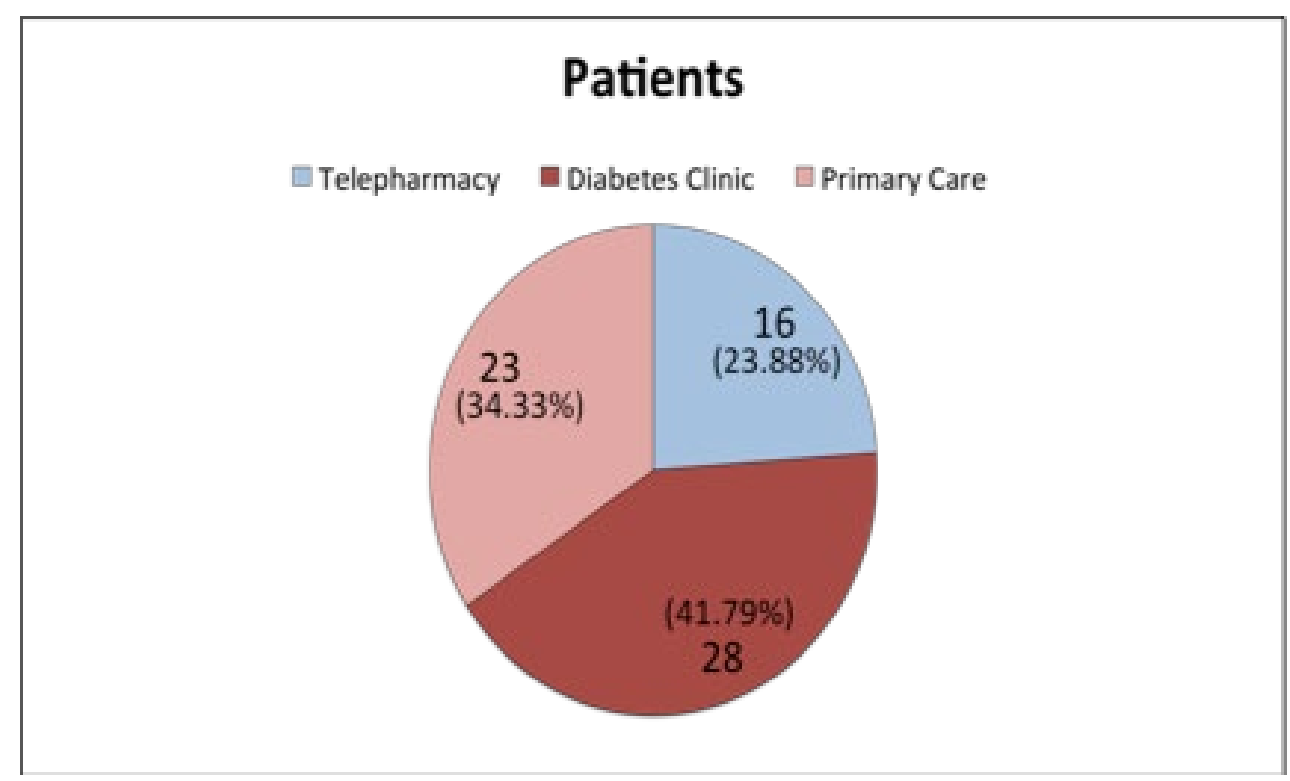

Figure 3. Percent change in A1c from baseline

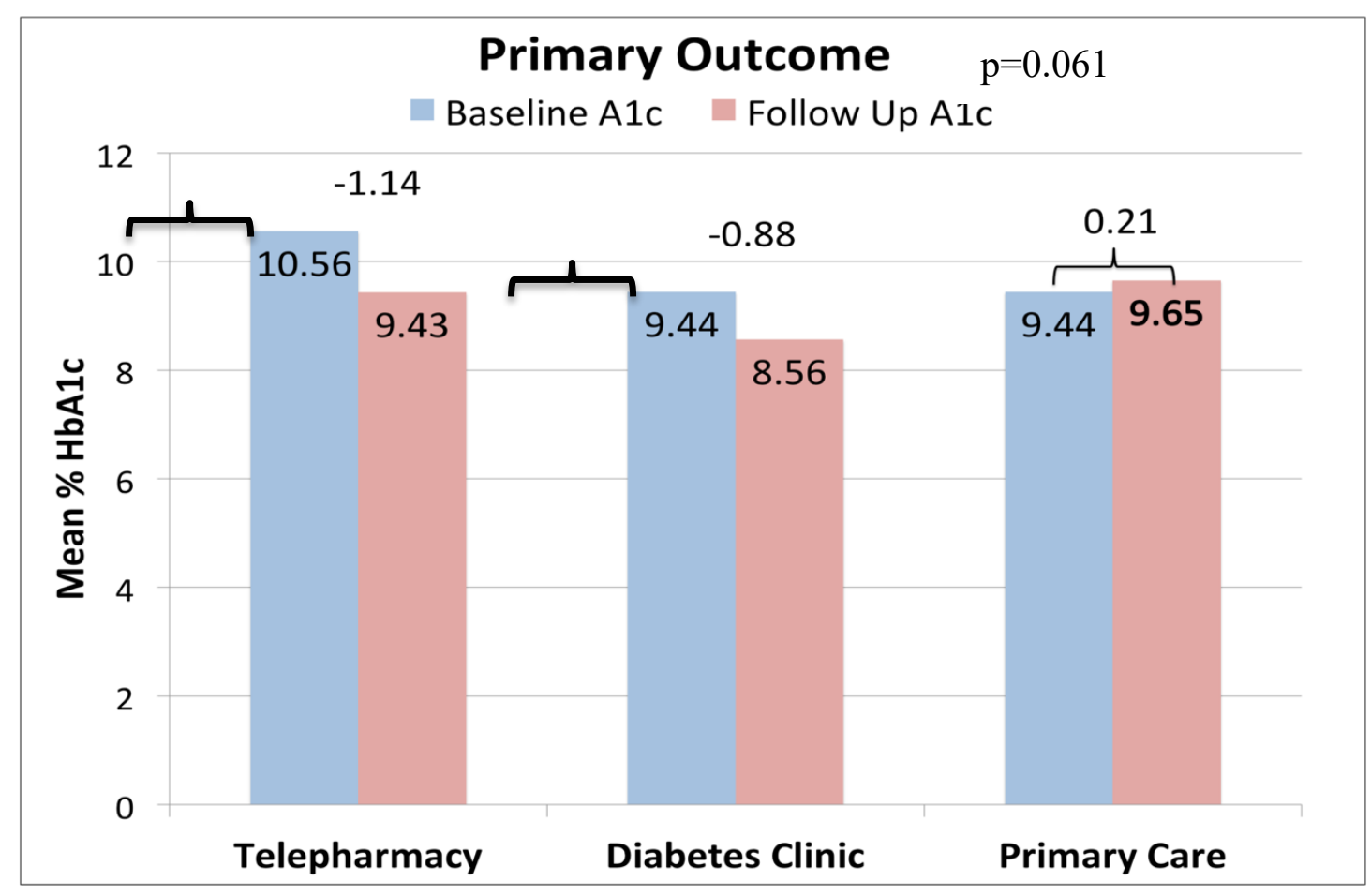


Table 1. Baseline patient characteristics

\begin{tabular}{|l|c|c|c|}
\hline & $\begin{array}{c}\text { Pharmacy Intervention } \\
(\mathrm{N}=16)\end{array}$ & $\begin{array}{c}\text { Diabetes } \\
\text { Clinic } \\
(\mathrm{N}=28)\end{array}$ & $\begin{array}{c}\text { Primary } \\
\text { Care } \\
(\mathrm{N}=23)\end{array}$ \\
$\mathrm{n}(\%)$ & $\mathrm{n}(\%)$ & $\mathrm{n}(\%)$ \\
\hline Gender (female) & $8(50)$ & $14(50)$ & 56.4 \\
\hline Average age (in years) & 55.8 & 55.04 & $21(91.3)$ \\
\hline Type 2 Diabetes & $15(93.75)$ & $22(78.6)$ & 32.74 \\
\hline Average BMI $\left(\mathrm{kg} / \mathrm{m}^{2}\right)$ & 31.54 & 33.06 & $17(73.91)$ \\
\hline $\begin{array}{l}\text { On oral diabetic } \\
\text { medication }\end{array}$ & $7(43.75)$ & $16(57.14)$ & $0(0)$ \\
\hline $\begin{array}{l}\text { Non-insulin injectable } \\
\text { therapy }\end{array}$ & $1(6.25)$ & $2(7.14)$ & \\
\hline
\end{tabular}

Table 2. Study Secondary Outcomes

\begin{tabular}{|c|c|c|c|c|}
\hline & $\begin{array}{l}\text { Pharmacy Intervention } \\
(\mathrm{N}=16) \\
\mathrm{n}(\%)\end{array}$ & $\begin{array}{c}= \\
\text { Diabetes Clinic } \\
(\mathrm{N}=28) \\
\mathrm{n}(\%)\end{array}$ & $\begin{array}{l}\text { Primary Care } \\
\qquad(\mathrm{N}=23) \\
\mathrm{n}(\%)\end{array}$ & $\mathrm{p}$-value \\
\hline $\begin{array}{l}\text { Patients experiencing at least a } 1 \% \mathrm{~A} 1 \mathrm{c} \\
\text { reduction from baseline during study } \\
\text { period }\end{array}$ & $7(43.75)$ & $10(35.71)$ & $6(26.09)$ & 0.510 \\
\hline \multicolumn{5}{|c|}{ Compliance with American Diabetes Association (ADA) Recommendations } \\
\hline Routine HbA1c Testing & $11(68.75)$ & $15(53.57)$ & 4 (17.39) & 0.003 \\
\hline $\begin{array}{l}\text { HbA1c reduced to general goal of less } \\
\text { than } 7 \%\end{array}$ & $0(0)$ & $4(14.29)$ & $3(13.04)$ & 0.290 \\
\hline
\end{tabular}

